\title{
Dynamic Modeling and Gait Analysis for Miniature Robots in the Absence of Foot Placement Control
}

\author{
Mohammad Askari and Onur Özcan \\ Bilkent University, Mechanical Engineering Department, Ankara, Turkey
}

\begin{abstract}
The study of animals and insects have led to realization that animals select their gaits, patterns of leg movement, according to speed. For proper gait planning, the legs must be controlled for proper foot placement with respect to the body motion and ground interactions. However, in small scale robotic platforms gait planning through foot placement control is neither cost effective nor easily attainable due to a lack of available sensors. Thus, even though a desired gait is envisioned at the design phase, it is not known whether the gait is optimum. In this work, we present the comprehensive dynamic model of the miniature foldable robot, MinIAQ-II, which has four independently actuated legs. Dynamic model is used to perform gait analysis, to investigate the difference between the intended gait and the achieved gait in the absence of foot placement control. The model is verified through slow speed walking experiments on flat terrain. The work presented can be modified for different miniature robots with passive legs to predict their locomotion under no foot placement control.

Index Terms-Origami-Inspired Robots, Foldable Robots,
\end{abstract} Miniature Robots, Legged Robots, Gait Analysis, Dynamics.

\section{INTRODUCTION}

Miniature or micro robotic platforms are good candidates for accomplishing tasks such as inspection, surveillance, and hazardous environment exploration where conventional macro robots fail to serve [1], [2], [3]. Such applications require these robots to potentially traverse uneven terrain, implying legged locomotion to be suitable for their design. The inspiration source of these legged robots, animals, modify their gaits with respect to their locomotion speeds or terrain characteristics [4], whereas robots, especially small ones, tend to work with a single gait set during the design phase. These miniature robots work very well, however unlike their biological inspirations, they cannot adapt their gaits and potentially they locomote in a sub-optimized manner. In addition, the lack of miniature robots that can modify their gaits lead to very few research on gait analysis in miniature robots [5]. Robots with higher number of active degrees of freedom can enable gait adjustment but are often avoided in small scale systems to minimize cost and complexity [6]. The second version of our robot, miniature quadruped with individually-actuated legs (MinIAQ-II) is designed with independent leg actuation to study locomotion under different gaits on a small robotic platform. [7].

MinIAQ-II (shown in Fig. 1) is a miniature, foldable quadruped that is 12-cm-long, 4.5-cm-high, and 6-cm-wide. It is made of a single sheet of cellulose acetate, has one DC motor for each leg, carries all the required electronics and a small Li-Po battery and yet weighs 23 grams. Its leg design is a compliant, one DOF fourbar mechanism with

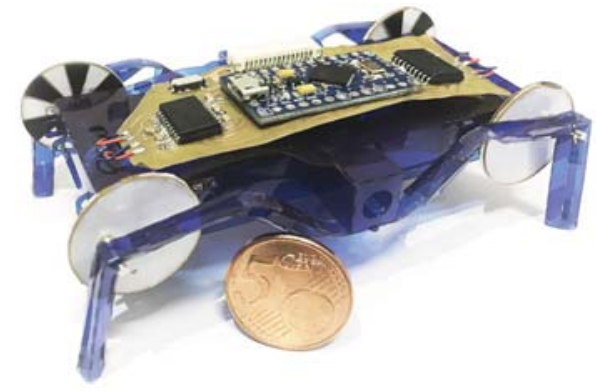

Fig. 1. MinIAQ-II: $23 \mathrm{~g}$ foldable quadruped with independent leg actuation.

an optimized trajectory. Its motors do not have encoders, so custom made absolute encoders were designed to control the gait of the robot. However, since the sensor feedback provides the relative position of the feet with respect to the body, the gait achieved is not the same as the gait intended due to the body rotations. A good solution to this issue would be implementing force or touch sensors at the feet so that foot placement control can be implemented. However, this is both infeasible and impractical for smallscale robots where production cost, payload capacity, power, and availability of proper sensing devices are all very limited. In this work, we modeled the dynamics of our miniature robot in a comprehensive way in order to show this difference between the achieved gait and the intended/ideal gait.

Many researchers have investigated the underlying dynamics in different kinds of animals and insects [8], [9], [10], and many others have been exploiting their findings to design respective bio-inspired walking [1], [2], [3] and crawling [11], [12] miniature robots. Most of these works are templates to predict dynamic behavior of simple modules of particular robot types, and less describe the full body dynamics with a more complete realistic model [8]. Furthermore, for the sake of simplicity, many of the existing models describe the dynamics in sagittal- or horizontal-plane and avoid complex 3D kinematics and transformations [13], [14]. However, for a robot with independent leg actuation and an aim to perform gait analysis, a comprehensive 3D model can give better insight into body rotations and out-of-plane locomotion.

The first and foremost step in dynamic modeling of a legged robot is to develop a mathematical model to predict its legs dynamics. One of the most commonly used leg models is the Spring Loaded Inverted Pendulum (SLIP) leg template [15]. There are several robots in the literature, from two (or more) DOF hopping and running simple robots [16], [17] to as high as a 20-DOF complex robot [18], that are 
modeled and controlled by SLIP. The SLIP leg template can best describe planar open-loop legs with active joints or single-link direct-drive legs as in a miniature robot like DASH [1] or in a large-scale robot like RHex [16]. However, for a robot leg that is driven by 1-DOF fourbar linkage with passive joints, SLIP model is relatively difficult to implement because the physical properties of the fictitious spring would be dependent on both material properties and the kinematics of the compliant mechanism, let alone modeling of feet slipping effects and predicting the unknown constrained stance and flight phases of the legs. We believe, for miniature robots like MinIAQ, modeling the legs by kinematic analysis of closed-chain mechanisms with well-defined planar and spatial constraints is a better approach.

In this work, we present a comprehensive dynamic model of our robot MinIAQ-II. We combined fourbar kinematics, rigid body dynamics, ground impact models, and friction models to acquire a 3D full body model of a miniature quadruped robot with modifiable gait. This model is able to accurately predict trends in locomotion on flat terrain. The contributions of this work are the comprehensive model of an underactuated robot with passive closed kinematic-chain legs and the analysis done using the model that shows how much the achieved gait is different than the intended gait.

\section{MinIAQ-II DESIGN AND CONTROL}

MinIAQ-II (see Fig. 1) is an origami-inspired foldable robot that is cut and folded from a single A4 size cellulose acetate sheet [7]. The motors used in the robot do not have any feedback sensors attached. Therefore, low cost, very low resolution absolute encoders are designed and cut for the motors to estimate leg position. Low-speed trot gait is selected as MinIAQ's primary form of locomotion. To achieve gait synchronization, two separate control loops, one for speed and the other for phase between the legs, are used. The gait is controlled by carefully selecting the relative order of the frequency gains to the phase control gains. Using such a controller, experiments showed that the phase of a leg can be controlled down to a phase error of about $20^{\circ}$. On the other hand, controlling the phases of the legs still does not guarantee that a specified gait will be achieved due to the body rotations; hence robot's dynamic behavior should be modeled to understand and estimate how gait changes with respect to the phase difference pattern between the legs.

\section{Modeling OF Robot Dynamics}

The following assumptions are used for this model:

1) Legs are modeled as fourbar linkages with rigid links and ideal pin joints, with care for the link shapes. The legs are considered to remain always parallel to the body and retain their shapes during contact.

2) The surface is modeled as a solid flat terrain and the feet make contact with the ground at a single point, i.e. torsional friction at the feet is neglected.

3) Legs are taken to be massless and the entire mass of the robot is uniformly distributed within its main frame.
This is because each leg weighs about $0.2 \mathrm{~g}$, which is less than $1 \%$ of the robot's body mass.

4) The motors are assumed to be running at constant speed, keeping the relative phase offset between the legs corresponding to the desired type of gait.

\section{A. Leg Kinematic Analysis}

Figure 2 shows a schematic diagram of the actual folded leg of MinIAQ-II and its generalized fourbar model, taking into account the coupler link shape. Since the mechanism is planar and is assumed to remain always parallel to the body frame, the kinematic equations are written with respect to a body-fixed coordinate frame. This body-attached frame is denoted by subscript $\mathcal{B}:\left\{X_{\mathcal{B}}, Y_{\mathcal{B}}, Z_{\mathcal{B}}\right\}$ throughout the paper and is located at the centroid (also the center of mass) of the main frame, inline with the motors.

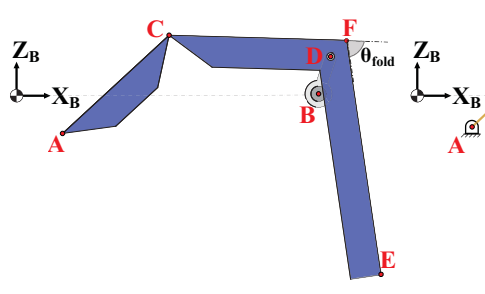

(a)

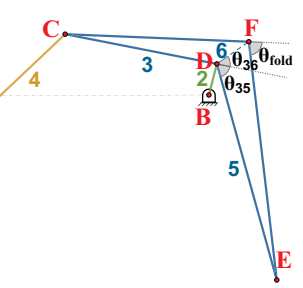

(b)
Fig. 2. (a) A representation of the actual folded leg shape of MinIAQ-II. (b) A schematic diagram of MinIAQ's generalized fourbar leg mechanism with geometrical consideration for the coupler link.

Table I lists the constant link lengths and fixed-angles shown in Fig. 2(b). $l_{k}$ denotes the length of the $k^{t h}$ link, and $\theta_{k}$ is used to represent the angle of each link, measured about $Y_{\mathcal{B}}$ axis. Note that links 5 and 6 are auxiliary links that are used to determine the position of the coupler point $E$ (tip of the foot). The position analysis of the $A B C D$ fourbar leads to determination of planar coordinates of joints $C$ and $D$. Then, by considering the $C D F E$ knee-shaped link geometry defined by the constants in the table, the coordinates of nodes $F$ and $E$ can be found in $X_{\mathcal{B}} Z_{\mathcal{B}}$ plane.

TABLE I

CONSTANT KinEMATIC PARAMETERS FOR MinIAQ-II
\begin{tabular}{cccc}
\multicolumn{4}{c}{} \\
\hline$l_{2}(\mathrm{~mm})$ & $l_{3}(\mathrm{~mm})$ & $l_{4}(\mathrm{~mm})$ & $l_{5}(\mathrm{~mm})$ \\
5.10 & 21.21 & 18.85 & 29.15 \\
\hline$l_{6}(\mathrm{~mm})$ & $\theta_{35}(\mathrm{deg})$ & $\theta_{36}(\mathrm{deg})$ & $\theta_{\text {fold }}(\mathrm{deg})$ \\
2.96 & 69.5 & 53.4 & 80 \\
\hline
\end{tabular}

Since the model aims to study gaits, the relative phase difference between the crank angles determine the type of gait. Therefore, the crank angle $\left(\theta_{2}\right)$ of each leg is defined as a function of time $(t)$, the constant angular speed of the motor $\left(\omega_{2}\right)$, and an initial phase $\left(\beta_{0}\right)$ corresponding to the desired gait phase offset between the legs. For a set of input crank angles at any given time, the solution to kinematic position analysis of the fourbar, previously reported in [7], yields planar coordinates of the joints on each leg. These planar coordinates can simply be turned into $3 \mathrm{D}$ position vectors representing the spatial coordinates of the joints with respect to the body-attached frame (see Fig. 3). 


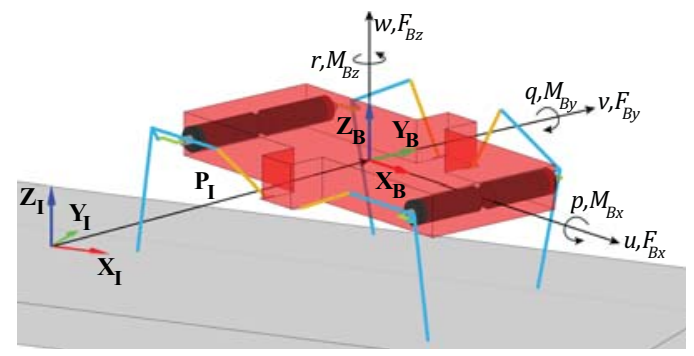

Fig. 3. A schematic representation of the Body-attached and the Inertial (global or world) reference frames used in deriving the dynamic equations.

If we denote the positions of the joints in the body-fixed frame by $\mathbf{r}_{\mathbf{k}}$, where $k=\{A, B, C, D, E, F\}$ corresponds to the nodes names in Figure 2, the joints velocity vectors (denoted by $\mathbf{v}_{\mathbf{k}}$ ) can be determined by kinematic velocity analysis. As the motors are assumed to turn at constant frequencies of $f_{j}$, the rotational velocity of the crank links in body-fixed frame are given by $\boldsymbol{\omega}_{\mathbf{2}_{\mathbf{j}}}=\left[\begin{array}{lll}0 & 2 \pi f_{j} & 0\end{array}\right]^{\top}$. The subscript $j=\{1,2,3,4\}$ indicates the motor or leg number, emphasizing the fact that each motor has its own degree of freedom. The linear speed of joint $D$ is given by:

$$
\mathbf{v}_{\mathrm{D}}=\mathbf{v}_{\mathrm{B}}+\omega_{2} \times \mathbf{r}_{\mathrm{BD}},
$$

where $\mathbf{v}_{\mathbf{B}}=\mathbf{0}$ as the motors are fixed within the body and $\mathbf{r}_{\mathrm{BD}}=\mathbf{r}_{\mathrm{D}}-\mathbf{r}_{\mathrm{B}}$. To calculate the speed of joint $C$, the rotational speed of link 3 or 4 is required:

$$
\begin{aligned}
& \mathbf{v}_{\mathbf{C}}=\mathbf{v}_{\mathbf{D}}+\omega_{3} \times \mathbf{r}_{\mathbf{D C}} \\
& \mathbf{v}_{\mathbf{C}}=\mathbf{v}_{\mathbf{A}}+\omega_{4} \times \mathbf{r}_{\mathrm{AC}},
\end{aligned}
$$

where $\mathbf{v}_{\mathbf{A}}=\mathbf{0}$ as $A$ is fixed to the body. Subtracting gives:

$$
\mathbf{v}_{\mathbf{D}}+\omega_{\mathbf{3}} \times \mathbf{r}_{\mathbf{D C}}-\boldsymbol{\omega}_{\mathbf{4}} \times \mathbf{r}_{\mathrm{AC}}=0
$$

which can be solved to determine links 3 and 4 rotational speeds, $\boldsymbol{\omega}_{3}$ and $\boldsymbol{\omega}_{4}$, and accordingly find the speed of joint $C, \mathbf{v}_{\mathbf{C}}$. Finally, the linear speeds of the tip of the foot $E$ and the fixed-fold node $F$ can be determined by,

$$
\begin{aligned}
& \mathbf{v}_{\mathbf{E}}=\mathbf{v}_{\mathbf{D}}+\omega_{3} \times \mathbf{r}_{\mathrm{DE}} \\
& \mathbf{v}_{\mathbf{F}}=\mathbf{v}_{\mathbf{D}}+\omega_{3} \times \mathbf{r}_{\mathbf{D F}} .
\end{aligned}
$$

Note that to determine the feet-ground contact, the only relevant point is $E$. The body-frame position and velocity vectors of the other nodes have no effect on robot dynamics.

\section{B. Robot Body Dynamics}

For a rigid body in a three dimensional Cartesian space, Lagrange's equations require local parameterization for the configuration space to define rotations. Such derivation of the equations of motion would hold locally as singularities can appear at certain configurations [19]. To derive the dynamics of MinIAQ in Cartesian space, the classical Newton-Euler method is utilized. The notation used in representing the Euler angle transformations and defining the vector quantities are in accordance with the aerodynamics conventions [20].

Two sets of coordinate systems are chosen to describe the motion of the robot as shown in Fig. 3, an inertial (global or world) reference frame fixed in space (denoted by $\left.\mathcal{I}:\left\{X_{\mathcal{I}}, Y_{\mathcal{I}}, Z_{\mathcal{I}}\right\}\right)$ and a body-attached frame moving and rotating with the robot (denoted by $\mathcal{B}:\left\{X_{\mathcal{B}}, Y_{\mathcal{B}}, Z_{\mathcal{B}}\right\}$ ).
The convention of placing the body-fixed axes at the center of mass, aligned with the principal axes of the frame, simplifies the moment equations by making the inertia tensor constant and diagonal. Thus, the equations of motion are developed in the body frame.

The six degrees of freedom in the body-fixed frame correspond to translation along and rotation about the body axes, denoted by $\mathbf{V}_{\mathcal{B}}$ and $\Omega_{\mathcal{B}}$, respectively:

$$
\mathbf{V}_{\mathcal{B}}=\left[\begin{array}{lll}
u & v & w
\end{array}\right]^{\top} \quad, \quad \boldsymbol{\Omega}_{\mathcal{B}}=\left[\begin{array}{lll}
p & q & r
\end{array}\right]^{\top} .
$$

The sum of all external forces $\mathbf{F}_{\mathcal{B}}$ and moments $\mathbf{M}_{\mathcal{B}}$ about the body axes, as shown in Fig. 3, are represented by, $\mathbf{F}_{\mathcal{B}}=\left[\begin{array}{lll}F_{\mathcal{B} x} & F_{\mathcal{B} y} & F_{\mathcal{B} z}\end{array}\right]^{\top}, \mathbf{M}_{\mathcal{B}}=\left[\begin{array}{lll}M_{\mathcal{B} x} & M_{\mathcal{B} y} & M_{\mathcal{B} z}\end{array}\right]^{\top}$.

Using Newton-Euler formulation, the translational equations of motions in the body frame can be written. Newton's second law $\left(\sum \mathbf{F}=m \dot{\mathbf{V}}\right)$ is only valid if $\mathbf{F}$ and $\mathbf{V}$ are defined in an inertial coordinate system. As a result, the Coriolis theorem must be utilized to determine the absolute time rate of change of a moving vector [19]. Therefore, by Newton's second law and Coriolis theorem, we have:

$$
\dot{\mathbf{V}}_{\mathcal{B}}=\frac{1}{m} \mathbf{F}_{\mathcal{B}}-\left(\boldsymbol{\Omega}_{\mathcal{B}} \times \mathbf{V}_{\mathcal{B}}\right)
$$

where $m$ is the constant mass of the robot. Equation (7) is a first-order vector ordinary differential equation (ODE) describing the time rate of change of linear velocity of the robot in the inertial space measured along the instantaneous body-fixed frame. The ODE can also be expanded to:

$$
\left(\begin{array}{c}
\dot{u} \\
\dot{v} \\
\dot{w}
\end{array}\right)=\frac{1}{m}\left(\begin{array}{l}
F_{\mathcal{B} x} \\
F_{\mathcal{B} y} \\
F_{\mathcal{B} z}
\end{array}\right)-\left(\begin{array}{c}
q w-r v \\
r u-p w \\
p v-q u
\end{array}\right) .
$$

Using similar steps for rotation, the absolute time rate of change of the inertial angular velocities, but decomposed to the body-fixed reference frame, can be determined from:

$$
\mathbf{M}_{\mathcal{B}}=\frac{\mathrm{d}\left(\mathbf{I}_{\mathrm{G}} \boldsymbol{\Omega}_{\mathcal{B}}\right)}{\mathrm{d} t}+\boldsymbol{\Omega}_{\mathcal{B}} \times\left(\mathbf{I}_{\mathrm{G}} \boldsymbol{\Omega}_{\mathcal{B}}\right),
$$

where $\mathbf{I}_{\mathbf{G}}$ is the mass moment of inertia tensor that should be calculated with respect to the body-fixed coordinate axes. For a rectangular prism (MinIAQ's rigid body), the inertia tensor reduces to a diagonal matrix where its diagonal elements are calculated by the $I_{i i}=\frac{m}{12}\left(\Delta j^{2}+\Delta k^{2}\right)$ formula. Here, $i$, $j$, and $k$ become $x, y$, or $z$ directions, depending on which axis the inertia is calculated about. The dimensions of the rectangular prism are $88 \mathrm{~mm}, 36 \mathrm{~mm}$, and $10 \mathrm{~mm}$ along $X_{\mathcal{B}}$, $Y_{\mathcal{B}}$, and $Z_{\mathcal{B}}$ axes, respectively. Since the inertia tensor is constant, Eq. 9 can be written as a first-order vector ODE:

$$
\left(\begin{array}{c}
\dot{p} \\
\dot{q} \\
\dot{r}
\end{array}\right)=\left(\begin{array}{l}
\left(M_{\mathcal{B} x}-\left(I_{z z}-I_{y y}\right) q r\right) / I_{x x} \\
\left(M_{\mathcal{B} y}-\left(I_{x x}-I_{z z}\right) r p\right) / I_{y y} \\
\left(M_{\mathcal{B} z}-\left(I_{y y}-I_{x x}\right) p q\right) / I_{z z}
\end{array}\right) .
$$

For determination of forces as well solving the system of ODEs, the robot's position and orientation in the inertial frame $\mathcal{I}:\left\{X_{I}, Y_{I}, Z_{I}\right\}$ must be known. The position of the robot's body centroid at any instance is given by the vector:

$$
\mathbf{P}_{I}=\left[\begin{array}{lll}
P_{I x} & P_{I y} & P_{I z}
\end{array}\right]^{\top} .
$$

$\mathbf{P}_{I}$ defines the translational vector between the two reference 
frames, and to define the rotations, a common approach is to use the Euler angles: roll $\phi$, pitch $\theta$, and yaw $\psi$. Roll corresponds to rotation about $X_{\mathcal{B}}$, pitch is rotation about $Y_{\mathcal{B}}$, and yaw is the rotation about $Z_{\mathcal{B}}$ axes (Fig. 3). Therefore, at any instance, the robot can be defined by position vector $\mathbf{P}_{I}$ and the orientation vector $\boldsymbol{\Gamma}$, where:

$$
\boldsymbol{\Gamma}=\left[\begin{array}{lll}
\phi & \theta & \psi
\end{array}\right]^{\top} \text {. }
$$

The orientation of the body is expressed in terms of yawpitch-roll (Z-Y-X) rotation angle sequence, according to [20]. Any vector defined in the inertial frame can be rotated to the body frame by multiplying it with a rotation matrix:

$$
\mathbf{R}_{I \rightarrow \mathcal{B}}=\mathbf{R}_{\phi} \mathbf{R}_{\theta} \mathbf{R}_{\psi} .
$$

On the other hand, the reverse is generally more desired where a vector defined in the body-fixed frame is to be moved to the inertial coordinate axes. Since $\mathbf{R}_{I \rightarrow \mathcal{B}}$ is an orthogonal rotation matrix, its inverse is equal to its transpose. The position vector $\mathbf{P}_{I}$ is defined in the inertial frame; therefore, the body-attached translational velocity $\mathbf{V}_{\mathcal{B}}$ should also be expressed in the inertial frame to write the position ODE as follows:

$$
\dot{\mathbf{P}}_{I}=\mathbf{R}_{\mathcal{B} \rightarrow I} \mathbf{V}_{\mathcal{B}}
$$

This can also be represented as three separate ODEs as:

$$
\left[\begin{array}{c}
\dot{P}_{I x} \\
\dot{P}_{I y} \\
\dot{P}_{I z}
\end{array}\right]=\left[\begin{array}{ccc}
c \theta c \psi & s \theta s \phi c \psi-s \psi c \phi & s \theta c \phi c \psi+s \psi s \phi \\
c \theta s \psi & s \theta s \phi s \psi+c \psi c \phi & s \theta c \phi s \psi-c \psi s \phi \\
-s \theta & c \theta s \phi & c \theta c \phi
\end{array}\right]\left[\begin{array}{c}
u \\
v \\
w
\end{array}\right],
$$

where $c$ and $s$ are abbreviations for cos and $\sin$, respectively. To derive the ODE expressing the rate of change of the Euler angles $(\dot{\boldsymbol{\Gamma}})$, they should be related to the body-fixed angular velocity vector $\Omega_{\mathcal{B}}$. This can be done by resolving the Euler rates into the body-fixed frame. Such mapping yields the three distinct orientation ODEs:

$$
\left(\begin{array}{c}
\dot{\phi} \\
\dot{\theta} \\
\dot{\psi}
\end{array}\right)=\left(\begin{array}{c}
p+(q \sin \phi+r \cos \phi) \tan \theta \\
q \cos \phi-r \sin \phi \\
(q \sin \phi+r \cos \phi) / \cos \theta
\end{array}\right) .
$$

Note that the singularity for a pitch of $\theta=90^{\circ}$ cannot happen under normal running conditions for a legged robot.

\section{Ground Interaction Forces}

In order to solve for the system of ordinary differential equations, the input to the system, which is the net external force and moment in the body-attached frame, must be determined. However, the characterization of the ground reaction forces generated due to contact of the feet requires analysis within the inertial reference frame. In this work, we used the model presented by Hunt and Crossley [21]. Hunt and Crossley's model represents the contact force by a nonlinear viscous-elastic spring damper element defined by:

$$
F_{N}=K \delta^{n}+D \dot{\delta}
$$

where $F_{N}$ is the normal contact force, $K$ denotes the generalized spring stiffness, $n$ is the nonlinear exponent, $D$ represents the damping coefficient, and $\delta$ and $\dot{\delta}$ are the deformation/penetration depth and rate, respectively. In MinIAQ, the analysis of the contact begins with determination of the foot position in the global frame to check whether the foot has penetrated into the ground, as shown in Fig. 4. The impact and friction forces are also related to the rate of foot penetration and sliding speed, respectively. The inertial velocity of the legs along the body-attached axes are estimated by the leg kinematic analyses and the speed of the robot. Since in derivation of the feet positions and speeds, the orientation, position, translation, and rotational velocity of the robot appears, it can be inferred that the ground reaction forces are implicit functions of all system states.

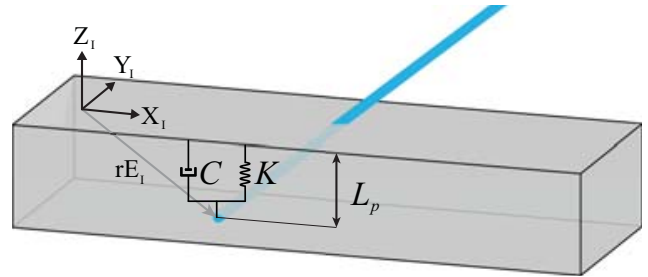

Fig. 4. Close-up schematic of the interpenetration between a foot and the ground during contact. Analysis of the impact must be done in the inertial axis, leading to the estimation of the normal force along $Z_{I}$, and analysis of the friction is done in $X_{I} Y_{I}$ plane.

For a leg penetrated into the ground, a normal force is generated as a function of a nonlinear spring stiffness $(K)$ and displacement based damping $(C)$.

$$
F_{N}= \begin{cases}0 & L_{p} \leq 0 \\ K\left(L_{p}\right)^{e}-C V_{p} & L_{p}>0\end{cases}
$$

where $C=\operatorname{STEP}\left(L_{p}, 0,0, d, c_{\max }\right) . L_{p}$ is the penetration depth, and $V_{p}$ is the absolute foot penetration rate. $K$ is the stiffness of the contact boundary and must not be confused with the stiffness of the leg, $e$ is the nonlinear force exponent, $c_{\max }$ is the maximum damping coefficient, and $d$ is the smallest penetration depth at which maximum damping is applied. The $\operatorname{STEP}\left(x, x_{1}, y_{1}, x_{2}, y_{2}\right)$ function is a smooth cubic approximation to a step of a random variable $x$, rising at $\left(x_{1}, y_{1}\right)$ and leveling off at $\left(x_{2}, y_{2}\right)$ [22].

The material used to fabricate MinIAQ, cellulose acetate sheets, is not a common structural material and its mechanical properties are not reported in literature. Thus, the selected parameters are taken from SolidWorks for a similar plastic material. In addition, it is suggested to select a force exponent of $e>1.5$ for better numerical convergence, a maximum damping such that $10^{-3} K<c_{\max }<10^{-4} K$, and the $d$ value as $0.01<d<0.1 \mathrm{~mm}$. The selected dynamic properties for the impact model are tabulated in Table II.

TABLE II

Force Model Parameters Used in Simulation of MiniaQ-II.

\begin{tabular}{cccc}
\hline$K\left(\mathrm{~N} / \mathrm{mm}^{e}\right)$ & $e$ & $c_{\max }(\mathrm{N} . \mathrm{s} / \mathrm{mm})$ & $d(\mathrm{~mm})$ \\
1150 & 2.0 & 0.588 & 0.1 \\
\hline$\mu_{s}$ & $\mu_{d}$ & $V_{s}(\mathrm{~mm} / \mathrm{s})$ & $V_{d}(\mathrm{~mm} / \mathrm{s})$ \\
0.4 & 0.3 & 1.0 & 10 \\
\hline
\end{tabular}

The friction component of the ground reaction force is represented in terms of a pseudo-Coulomb dry friction model given by [23]. This friction model assumes that pure stiction does not occur, but that the bodies in contact continuously move relative to each other at a negligibly small velocity for stiction phase. The direction of the friction force is defined based on the the relative movement of the feet on the ground 
and its magnitude depends on the pseudo friction coefficient, the normal impact force, and nonlinearly on the relative velocity. The pseudo-Coulomb friction coefficient denoted by $\mu$ is given by:

$$
\begin{aligned}
\mu= & \operatorname{STEP}\left(V_{\text {rel }},-V_{d}, \mu_{d},-V_{s}, \mu_{s}\right)+ \\
& \operatorname{STEP}\left(V_{r e l},-V_{s}, \mu_{s}, V_{s},-\mu_{s}\right)+, \\
& \operatorname{STEP}\left(V_{\text {rel }}, V_{s},-\mu_{s}, V_{d},-\mu_{d}\right)
\end{aligned}
$$

where $V_{\text {rel }}$ is the relative velocity between the feet and the ground, $\mu_{s}$ and $\mu_{d}$ are static and dynamic coefficients of friction, and $V_{s}$ and $V_{d}$ are static and dynamic transition velocities, respectively. By definition, $\mu_{s}$ and $\mu_{d}$ are $\in[0,1]$, typically $\mu_{s}>\mu_{d}$, and $V_{s}<V_{d}$. The tangential friction force is simply estimated by multiplying the pseudo coefficient $\mu$ with the impact force.

If we denote $\mathbf{r}_{\mathbf{E}_{I_{\mathbf{j}}}}$ and $\mathbf{v}_{\mathbf{E}_{I_{\mathbf{j}}}}$ by $\left[\begin{array}{lll}r_{1_{j}} & r_{2_{j}} & r_{3_{j}}\end{array}\right]^{\top}$ and $\left[\begin{array}{lll}v_{1_{j}} & v_{2_{j}} & v_{3_{j}}\end{array}\right]^{\top}$, respectively, then for each leg $L_{p_{j}}=-r_{3_{j}}$, $V_{p_{j}}=v_{3_{j}}$, and $V_{r e l_{j}}=\sqrt{v_{1_{j}}^{2}+v_{2_{j}}^{2}}$. Equations (15) and (16) are next calculated to determine the magnitude of the normal force $\left(F_{N_{j}}\right)$ and coefficient of friction $\left(\mu_{j}\right)$, for each foot. The contact force for each leg can be written in vector form with respect to the body-fixed frame as such:

$$
\mathbf{F}_{\mathcal{B}_{\mathbf{j}}}=\mathbf{R}_{I \rightarrow \mathcal{B}}\left(F_{N_{j}}\left[\begin{array}{l}
0 \\
0 \\
1
\end{array}\right]+\frac{\mu_{j} F_{N_{j}}}{V_{r e l_{j}}}\left[\begin{array}{c}
v_{1_{j}} \\
v_{2_{j}} \\
0
\end{array}\right]\right) .
$$

If we denote the weight force in the inertial frame by $\mathbf{W}_{I}=\left[\begin{array}{lll}0 & 0 & -m g\end{array}\right]^{\top}$, where $m$ is the robot's mass and $g$ is the gravitational acceleration, then the net external force and moment in the body-fixed frame can be evaluated by:

$$
\begin{aligned}
\mathbf{F}_{\mathcal{B}} & =\mathbf{R}_{I \rightarrow \mathcal{B}} \mathbf{W}_{I}+\sum_{j=1}^{4} \mathbf{F}_{\mathcal{B}_{\mathrm{j}}} \\
\mathbf{M}_{\mathcal{B}} & =\sum_{j=1}^{4} \mathbf{r}_{\mathbf{E}_{\mathcal{B}_{\mathrm{j}}}} \times \mathbf{F}_{\mathcal{B}_{\mathrm{j}}} .
\end{aligned}
$$

\section{Solving the Complete System of Equations}

The dynamics of the robot is given by a system of 12 highly nonlinear ordinary differential equations, describing the translation (Eq. 8), rotation (Eq. 10), position (Eq. 13), and orientation (Eq. 14) rates in either the bodyfixed axes or the inertial frame. If we denote the states by $\mathbf{X}=\left[\begin{array}{llll}\mathbf{P}_{\mathcal{I}}{ }^{\top} & \boldsymbol{\Gamma}^{\top} & \mathbf{V}_{\mathcal{B}}{ }^{\top} & \boldsymbol{\Omega}_{\mathcal{B}}{ }^{\top}\end{array}\right]^{\top}$, or equivalently:

$$
\mathbf{X}=\left[\begin{array}{llllllll}
P_{I x} & P_{I y} & P_{I z} & \phi \theta & \psi & u v w & p & q r
\end{array}\right]^{\top},
$$

then, $\dot{\mathbf{X}}$ can be represented as the twelve equations (Eq.s 8 , 10, 13, and 14) written underneath eachother. For a given initial state vector the solution to the system of ODEs can be obtained using a nonlinear solution technique. However, the standard numerical nonlinear methods, such as Euler or Runge-Kutta, can exhibit instability in the solutions. This is due to the sharp increase in the contact force at the instance of penetration of a foot into the ground. This behavior in ODEs is described as stiffness. The system of ODEs is formulated in MATLAB environment and a built-in stiff integration technique, ode $15 \mathrm{~s}$, is used to solve for the system. Utilizing a stiff solver (ode15s) in comparison to a non-stiff solver (ode 45), Runge-Kutta method, has shown to exhibit an increase in stability and a significant decrease in simulation time.

\section{MODEL VERIFICATION}

Using the dynamic model, multiple cases are run and compared with experimental data of the actual robot. Since MinIAQ-II is designed for trot gait, the experiments were run for trot gait straight locomotion and trot gait turning at $2.5 \mathrm{~Hz}$ motor frequency. The robot has no sensing device on board; therefore, the identification of the state variables is done using raw video shots of multiple runs. For straight line locomotion, the position of the approximate center of gravity and variations of pitch angle are estimated from the side views, and the rolling effect of the robot is tracked from frontal shots. In turning experiments, the videos are shot from top view, the yaw angle variation corresponding to turning speed of the robot is measured.

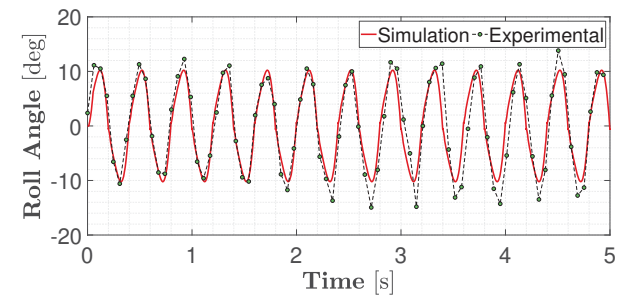

(a)

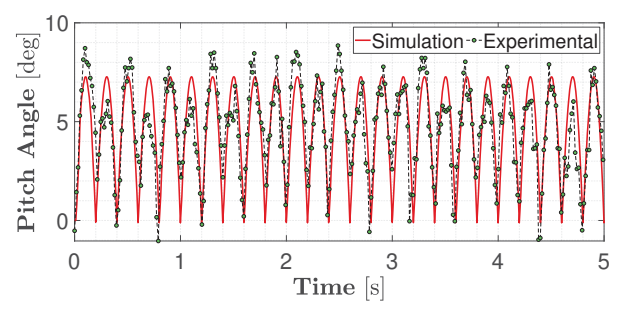

(b)

Fig. 5. Trot gait straight locomotion for MinIAQ-II at $2.5 \mathrm{~Hz}$ motor speed. (a) The roll angle comparison. (b) The pitch angle comparison.

Figures 5(a) and 5(b) show the results for roll and pitch angle variation over five seconds, respectively. The model estimates the rolling and pitching of MinIAQ-II during straight line trot locomotion reasonably well. Comparison of the simulation and experimental results shows that for half of a step cycle, the pitch is estimated better than for the other half. As implied by the symmetric periodicity trend in the simulated data, it is expected to observe a symmetric pitch variation in the experimental data as well, when right and left side legs alternate. Yet, the robot tends to fall onto one side during locomotion faster than it does on the other side. This behavior can be attributed to a shift in the center of gravity position in the body, due to assembly problems.

The results for the position of the robot over five seconds during straight locomotion are shown in Fig. 6. Since extracting the exact centroid location of the body was difficult from the videos, a point on the side but very close to the COG was tracked and the simulation results are also provided for the same point. The results shown are in considerable agreement with the experiments. The small difference between the 


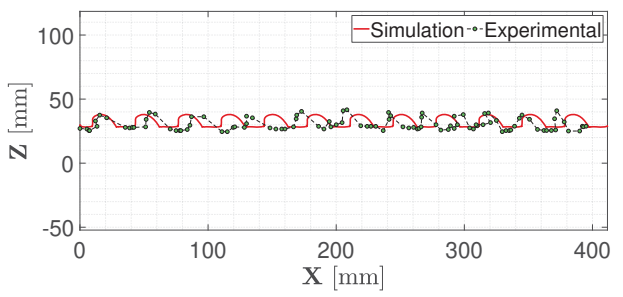

Fig. 6. Trot gait simulation, robot's trajectory at $2.5 \mathrm{~Hz}$ motor speed. simulation and the experimental results can be attributed to small errors in the estimated friction and impact parameters.

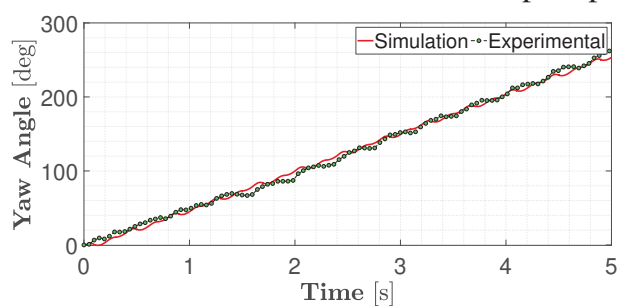

Fig. 7. Yaw angle variation during in-place turning at $2.5 \mathrm{~Hz}$ motor speed.

In performing zero radius turns, the experiments are carried out by reversing the direction of the motors on one side of the robot, while keeping the speeds of all constant. The speed of rotation in turning tests can simply be measured from the variations in yaw angle, presented in Fig. 7. The simulation results closely match with the extracted yaw angles and the nearly linear trends in the plots suggest that the robot is capable of performing "nearly" in-place turns at relatively constant speeds.

\section{GAIT ANALYSIS}

A comprehensive explanation to gaits is given by [24], where a gait is defined by "the time and the location of the placing and lifting of each foot, coordinated with the motion of the body in its six degrees of freedom, in order to move the body from one place to another". The key concept in this definition is the coordination of a gait with the body motion. Without this coordination, and without control on foot placement and/or realization of body movements, robot will be walking unplanned, and potentially with a different gait than intended. Since not many sensors are available for foot placement control in miniature robots like MinIAQ-II, the gaits of these robots are often not controlled.

In order to investigate how closely the actual gait matches with the intended gait, the validated trot case gait diagrams are presented for MinIAQ-II. In these diagrams (Figures 8 and 9), the length of each horizontal bar defines the duty factor, which is the duration that a leg is in contact with the ground over one stride cycle. A forward force is shown with green color and a backward force is shown by red. Each gait diagram represents an intended/ideal gait pattern along with an achieved/actual gait pattern. A robot should not drag its feet on the ground to generate a backward dissipative force, and that is why the ideal gait is represented by full green color. The gait diagram given in Fig. 8 shows the pattern for gaits for five seconds of simulation. The actual gait has a periodic trend per each stride, making examination of the results for a single cycle more practical and understandable.

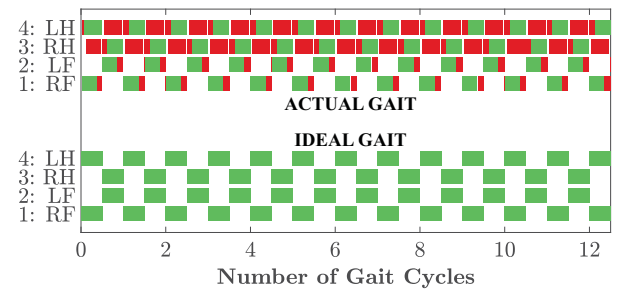

Fig. 8. A complete trot gait diagram for MinIAQ-II, for a simulation of five seconds at $2.5 \mathrm{~Hz}$, equivalent to 12.5 stride cycles. Since the actual gait is periodic in time, representation of a single cycle would be enough.

MinIAQ-II is originally designed to run with trot gait, where the diagonal pair of legs are in phase and the pairs have $180^{\circ}$ of phase offset with respect to each other [25]. But the robot does not conform to an ideal trot gait. As can be seen from the gait diagram (Fig. 9), the hind legs spend a lot of time being dragged on the surface as shown by red. Almost over the entire cycle, the robot is running on three legs, as opposed to the ideal trot gait where this is supposed to be only two at any instance. This observation suggests that the robot is behaving more like a walking gait pattern. This is also very close to our observations during the experiments.

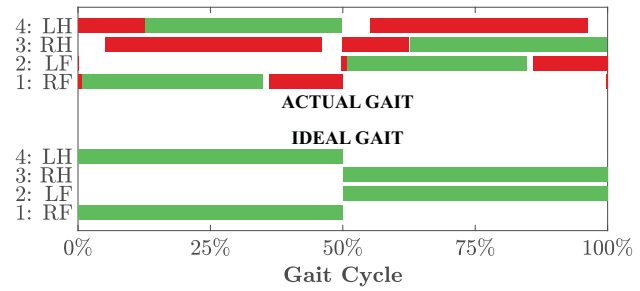

Fig. 9. A single cycle trot gait diagram for MinIAQ-II, simulated at a motor speed of $2.5 \mathrm{~Hz}$.

\section{CONCLUSiOn AND Future WORK}

In this work, we presented the comprehensive dynamic model of our foldable miniature robot, MinIAQ-II. The model is needed because the robot does not have touch sensors at the feet, preventing us from performing foot placement control; hence the robot cannot keep a steady gait. Dynamic model is developed step-by-step so that researchers working with similar robots can follow the model and modify it according to their needs. The model is then verified by comparing the simulation results with experiments.

The results presented in this work demonstrate how the intended and actual gaits can be different in a miniature robot where there is no control on foot placement. The model can even be used as a tool to seek for a desired gait pattern that can minimize the difference between the intended gait and the actual gait. The validated model can be utilized to systematically analyze and optimize gaits specific to a robot. However, this is considered as the future work for MinIAQ as the gait analysis of the robot is at its preliminary stage.

\section{ACKNOWLEDGMENTS}

The authors would like to thank Cem Karakadioglu for his invaluable contribution in robot design and experiments. This work is partially funded by the Scientific and Technological Research Council of Turkey (TÜBİTAK) grant no. 116E177. 


\section{REFERENCES}

[1] P. Birkmeyer, K. Peterson, and R. S. Fearing, "DASH: A dynamic 168 hexapedal robot," in Intelligent Robots and Systems, 2009. IROS 2009. IEEE/RSJ International Conference on. IEEE, 2009, pp. 2683-2689.

[2] O. Ozcan, A. T. Baisch, D. Ithier, and R. J. Wood, "Powertrain selection for a biologically-inspired miniature quadruped robot," in 2014 IEEE International Conference on Robotics and Automation (ICRA). IEEE, 2014, pp. 2398-2405.

[3] A. M. Hoover, E. Steltz, and R. S. Fearing, "RoACH: An autonomous 2.4 g crawling hexapod robot," in Intelligent Robots and Systems, 2008. IROS 2008. IEEE/RSJ International Conference on. IEEE, 2008, pp. 26-33.

[4] R. M. Alexander, "The gaits of bipedal and quadrupedal animals," The International Journal of Robotics Research, vol. 3, no. 2, pp. 49-59, 1984.

[5] R. S. Pierre and S. Bergbreiter, "Gait exploration of sub-2 g robots using magnetic actuation," IEEE Robotics and Automation Letters, vol. 2, no. 1, pp. 34-40, Jan 2017.

[6] M. Agheli, S. G. Faal, F. Chen, H. Gong, and C. D. Onal, "Design and fabrication of a foldable hexapod robot towards experimental swarm applications," in 2014 IEEE International Conference on Robotics and Automation (ICRA). IEEE, 2014, pp. 2971-2976.

[7] M. Askari, C. Karakadığlu, F. Ayhan, and O. Özcan, "MinIAQ-II: A miniature foldable quadruped with an improved leg mechanism," in 2017 IEEE International Conference on Robotics and Biomimetics (ROBIO). IEEE, 2017.

[8] P. Holmes, R. J. Full, D. Koditschek, and J. Guckenheimer, "The dynamics of legged locomotion: Models, analyses, and challenges," SIAM review, vol. 48, no. 2, pp. 207-304, 2006.

[9] D. I. Goldman, T. S. Chen, D. M. Dudek, and R. J. Full, "Dynamics of rapid vertical climbing in cockroaches reveals a template," Journal of Experimental Biology, vol. 209, no. 15, pp. 2990-3000, 2006.

[10] R. J. Full and M. S. Tu, "Mechanics of a rapid running insect: two, four-and six-legged locomotion," Journal of Experimental Biology, vol. 156 , no. 1 , pp. $215-231,1991$.

[11] K. Zhang, C. Qiu, and J. S. Dai, "Helical kirigami-enabled centimeterscale worm robot with shape-memory-alloy linear actuators," Journal of Mechanisms and Robotics, vol. 7, no. 2, p. 021014, 2015.

[12] C. D. Onal, R. J. Wood, and D. Rus, "An origami-inspired approach to worm robots," IEEE/ASME Transactions on Mechatronics, vol. 18 no. 2, pp. 430-438, 2013.

[13] M. M. Bohra and M. R. Emami, "An evolutionary approach to feline rover gait planning," in Robotics and Biomimetics (ROBIO), 2014 IEEE International Conference on. IEEE, 2014, pp. 767-772.

[14] K. L. Hoffman, "Design and locomotion studies of a miniature centipede-inspired robot," Doctoral dissertation, May 2013

[15] M. H. Raibert, Legged robots that balance. MIT press, 1986.

[16] U. Saranli, M. Buehler, and D. E. Koditschek, "RHex: A simple and highly mobile hexapod robot," The International Journal of Robotics Research, vol. 20, no. 7, pp. 616-631, 2001.

[17] A. Spröwitz, A. Tuleu, M. Vespignani, M. Ajallooeian, E. Badri, and A. J. Ijspeert, "Towards dynamic trot gait locomotion: Design, control, and experiments with cheetah-cub, a compliant quadruped robot," The International Journal of Robotics Research, vol. 32, no. 8, pp. 932 950, 2013.

[18] D. Gong, P. Wang, S. Zhao, L. Du, and Y. Duan, "Bionic quadruped robot dynamic gait control strategy based on twenty degrees of freedom," IEEE/CAA Journal of Automatica Sinica, vol. 5, no. 1, pp. 382-388, 2018

[19] M. W. Spong and M. Vidyasagar, Robot dynamics and control. John Wiley \& Sons, 2008.

[20] L. V. Schmidt, Introduction to aircraft flight dynamics. American Institute of Aeronautics and Astronautics, 1998.

[21] K. H. Hunt and F. R. E. Crossley, "Coefficient of restitution interpreted as damping in vibroimpact," Journal of applied mechanics, vol. 42, no. 2 , pp. 440-445, 1975.

[22] D. Negrut and A. Dyer, "Adams/solver primer," MSC. Software Documentation, Ann Arbor, 2004

[23] M. Wojtyra, "Multibody simulation model of human walking," $\mathrm{Me}$ chanics Based Design of Structures and Machines, 2003.

[24] S.-M. Song and K. J. Waldron, Machines that walk: the adaptive suspension vehicle. MIT press, 1989.

[25] D. Owaki and A. Ishiguro, "A quadruped robot exhibiting spontaneous gait transitions from walking to trotting to galloping," Scientific reports, vol. 7, no. 1, p. 277, 2017. 